\title{
APROXIMACIÓN A LA INTEGRACIÓN DEL ALUMNADO CON NECESIDADES EDUCATIVAS ESPECIALES EN LA UNIVERSIDAD
}

\section{AN APPROACH TO THE INTEGRATION OF STUDENTS WITH SPECIAL EDUCATIONAL NEEDS IN THE UNIVERSITY}

\author{
Tiberio Feliz Murias* y $M^{a}$ Carmen Ricoy Lorenzo** \\ UNED y Universidad de Vigo
}

\begin{abstract}
RESUMEN
Desde este trabajo asumimos la integración del alumnado con necesidades educativa especiales en la Universidad como un reto necesario que juntos hemos de compartir. Nos aproximamos a este ambicioso desafío, a través del análisis que realizamos en la Universidad, particularmente, en la UNED. El estudio abordado nos permite conocer las posibilidades que esta Universidad brinda, precisamente por darse en ella la enseñanza a distancia. En todo caso, y por supuesto valorando lo hecho hasta el momento, planteamos algunas mejoras o propuestas estructurales y normativas que aseguren, en mayor medida, la integración de las personas con necesidades educativas especiales en el ámbito universitario extensible al cotidiano.
\end{abstract}

Palabras clave: Universidad; Necesidades educativas especiales; diversidad.

\begin{abstract}
With this article, we are assuming the integration of students with special educational needs in the university as a necessary challenge we have to develop together. This approach is developed through the study of a special situation: the Spanish Distance University (UNED). By these means, we can know the possibilities this university offers, due to its distance systems. We value all the efforts they have done up to date but we suggest some improvements, and structural, normative proposals to assure the integration of persons with special educational needs in the university circles, trying to extend it to the diary life.
\end{abstract}

Key words: University; Special educational needs; Diversity.

* Es profesor del área de Didáctica y Organización Escolar en la Facultad de Educación de la UNED. Líneas de investigación: Recursos de formación; diversidad y necesidades educativas especiales; metodología y técnicas de investigación; educación de adultos. E-mail: tfeliz@edu.uned.es

** Es profesora del área de Didáctica y Organización Escolar en la Facultad de C.C. de la Educación de la Universidad de Vigo. Líneas de investigación: Recursos de formación; diversidad y necesidades educativas especiales; metodología y técnicas de investigación; educación de adultos. E-mail: cricoy@uvigo.es 


\section{Introducción: Contribuciones actuales sobre la temática}

Como sabemos las necesidades educativas especiales representan una parte de la diversidad educativa pero no su totalidad. Esto nos lleva a entrar someramente en una revisión terminológica. Es obvio, que en el momento actual ambos aspectos continúan preocupando a la ciudadanía y en particular a los educadores. Sin embargo, como aspectos terminológicamente comunes quisiéramos reseñar el carácter normalizador de los referidos vocablos. Asimismo, Peñafiel (2000) resalta que es la LOGSE de 1990 la que en nuestro país propicia un uso generalizado de los citados términos, impregnándole apariencia antidiscriminatoria.

Por su parte el principio de atención a la diversidad hace referencia a un conjunto de intervenciones formativas, que desde una oferta educativa semejante para todos los estudiantes ofrece respuestas diferenciadas a aquellos que las precisan ajustándolas a sus características (Aguilar, 2000). La atención a la diversidad puede requerir el uso de estrategias, metodología, medios, etc. diferenciados, específicos y/o complementarios, así como una planificación totalmente adaptada en determinados casos. Práctica que no tiene porque impedir el seguimiento de una enseñanza normalizada en la institución educativa (a este respecto también nos queda mucho por avanzar en el contexto universitario). La diversidad se manifiesta como un hecho diferenciador de origen natural o adquirido. Para Sánchez y Quiroga (1998) y López y Zafra (2003), se requieren respuestas educativas integradoras a través del uso de estilos y recursos de enseñanza-aprendizaje, específicos o/y adaptables a las necesidades individuales de cada alumno. Por fortuna las personas somos distintas las unas de las otras (cultural, biológica, social, conductualmente, etc.) y en ello hay una enorme riqueza huma.

Así mismo, las necesidades educativas especiales suponen, como decimos, una parte relevante de la diversidad humana. Como terminología fue introducida ya en el informe Warnock de 1978, dónde se recogían las iniciativas para la integración escolar y social de los discapacitados, inspirando posteriormente leyes de educación de diferentes países, entre los que se encuentra después de una década el nuestro con la LOGSE de 1990. Bautista (1993), ve en este cambio conceptual un avance significativo a nivel educativo, así como un medio que posibilita el paso de la discriminación a la integración y de la homogeneidad a la diversidad.

La terminología al uso de necesidades educativas especiales alude fundamentalmente a la respuesta específica que debe dar la educación al alumnado que precise medidas individuales en su formación, buscando a compensar de forma integradora dicha situación (Moya, Barrero y otros, 2000). En todo caso supone apoyos adicionales o diferenciados de los utilizados con los demás estudiantes dentro de la institución educativa, así como adaptaciones y/o complementos pedagógicos, servicios, materiales, etc. Ricoy y Feliz (2002), ven que el concepto está ligado al entorno formativo, entendiendo que las necesidades educativas especiales están referidas a las ayudas específicas de: acceso al currículum adaptándolo; atención especial al contexto social y clima emocional del alumno; servicios especializados; eliminación de barreras físicas; etc. La problemática que presenta el alumnado en su desarrollo puede ser por defecto o exceso y así darse de forma temporal o permanente. Este hecho supone un gran reto para la comunidad universitaria extensible a toda la ciudadanía, con el propósito de integrar y normalizar la vida de las personas, singularmente la de los discapacitados. 
Coincidimos con Aguilar (op. cit.), en que la calidad y equidad en la atención a las personas con discapacidad, tiene que ser una lucha de todos nosotros. El espacio universitario como tal centro de formación, cultura, ciencia, entretenimiento, etc. habrá de ser un referente comunitario ejemplar, convirtiéndose en un recinto abierto a las diferencias individuales. Cabe a todo, ni la legislación al respecto ni siquiera la literatura científica ha fructificado en retos que a la Universidad le quedan por afrontar. Como escaso referente, podemos citar el artículo 31 de la Ley de Integración Social de los Minusválidos (BOE, 1982), que indica en su segundo apartado dos medidas especialmente pensadas para los "minusválidos que cursen estudios universitarios":

- La ampliación del número de las convocatorias establecido con carácter general en la medida que compense su dificultad.

- La adaptación de las pruebas a las características de su minusvalía sin mengua del nivel exigido.

Éste es el escaso marco legislativo que tenemos en España sobre la integración de las personas discapacitadas en la Universidad. Es cierto que este nivel plantea unas consideraciones a las que son ajenas otros niveles de enseñanza pero también refleja la idea generalizada de que no se concibe como un espacio propio para personas discapacitadas. En otras palabras, los hechos vienen a plasmar que las referidas personas tienen difícil cabida en la enseñanza superior. Los motivos de esta concepción están a caballo de la imagen que tenemos en la Sociedad de la Enseñanza Superior y de la consideración sobre las posibilidades de formación de las personas discapacitadas: parecen extremos incompatibles o difíciles de conciliar. La primera se asocia a la capacidad intelectual; la segunda a su carencia. El problema está servido. La Institución Universitaria no estimula la participación suficientemente y las personas con discapacidad presentan una demanda escasa. Ésa es la realidad de una situación con muchos altibajos y fricciones que en la Educación a Distancia parecen presentar aún mayores dificultades: la falta de contacto, la soledad, el volumen de contenidos y alumnado, las evaluaciones presenciales, etc. Sin embargo, las cifras de la UNED parecen contradecir esa impresión inicial. Ello merece una profunda reflexión y análisis de la situación. No hemos de olvidar que el mismo concepto de integración conlleva el reconocimiento del derecho de todos los ciudadanos a la educación, cuya base legal en nuestro estado la recoge su carta magna (Constitución Española de 1978, artículo 27). Para Ricoy y Feliz (op. cit.), el objetivo último de la integración es la optimización del bienestar desde el desarrollo de las múltiples dimensiones del ser humano en una sociedad dinámica.

\section{Objetivos}

El objetivo general que nos planteamos en el presente estudio lo enunciamos de forma sintética del siguiente modo:

Aproximarnos al conocimiento de la realidad sobre la situación de la integración del alumnado con necesidades educativas especiales en la UNED.

Además, hemos trazado como objetivos más específicos los recogidos a continuación:

- Describir el marco institucional y normativo que posibilita la integración de alumnos/as con Necesidades Educativas Especiales en la UNED. 
- Conocer, barajar y contrastar datos recientes sobre el alumnado con necesidades educativas especiales, frente a la totalidad de estudiantes matriculados en esta Universidad.

- Analizar las dificultades que se encuentran a la hora de hacer efectiva esa integración.

- Realizar propuestas y/o sugerencias de mejora para asegurar los procesos de integración y normalización.

Como es obvio, podremos dar respuesta a dichos objetivos, en la medida en que los datos analizados nos faciliten la información suficiente y oportuna para ello.

\section{Metodología}

Se trata de un estudio descriptivo en el que analizamos los hechos, fundamentalmente a través de las cifras de alumnado matriculado en la UNED en un curso académico reciente. A la investigación descriptiva tal como refleja Best (1981) le concierne recoger prácticas, opiniones, hechos, sucesos, etc. que se dan en determinados contextos. Rebasando la mera presentación de datos configura un factor interpretativo sobre la magnitud, significado o importancia de lo que se describe. Para ello nos fue necesario en el trabajo aquí recogido organizar, sistematizar, examinar e interpretar con detenimiento los datos que nos ha facilitado la UNED.

En la misma línea de lo anterior también Fox (1987) considera que los datos descriptivos pueden aportarnos información para conocer en mayor medida las situaciones y circunstancias de los hechos a indagar. En este trabajo, nuestra pretensión como dijimos está en analizar con detenimiento el estado de la situación sobre la integración del alumnado con necesidades educativas especiales en la UNED. Aunque los datos descriptivos pueden ser cualitativos y cuantitativos, nosotros centramos la presentación de resultados en los de carácter cuantitativo a través de los descriptores de porcentaje y frecuencia, dado que los datos descriptivos de naturaleza cuantitativa facilitan el estudio de muestras grandes de sujetos y también el análisis con detenimiento de grupos más reducidos (Bisquerra, 1989; y García Llamas, 1994).

Básicamente, en el trabajo aquí aportado nos centramos en el examen de los datos sobre el alumnado matriculado en la UNED en el curso académico 2000/01. Con la información obtenida, analizamos las posibilidades de integración del alumnado discapacitado matriculado en la UNED, que, además, completamos con la recogida de experiencias y casos que justifican la necesidad de reforzar las medidas tendentes a la integración y normalización de los alumnos/as con Necesidades Educativas Especiales en la citada Universidad. A partir de lo dicho, proponemos alternativas de mejora.

\section{Presentación e interpretación de resultados}

\section{La participación en la UNED}

En el curso 1999-2000 se han matriculado 1.330 alumnos con discapacidades. En el curso siguiente, el 2000/2001, han sido 1.698 alumnos, un 29,66\% más. Habitualmente, el nú- 
mero de personas discapacitadas que estudian en las universidades presenciales es muy inferior. Esta diferencia se puede explicar considerando los siguientes factores:

- La Educación a Distancia elude los problemas de accesibilidad física con que cuentan aún muchos edificios tradicionales de la Universidad presencial.

- Ofrece una posibilidad óptima de organización y adaptación a los tiempos y disponibilidad personal.

- Garantiza la posibilidad de disponer de los textos y materiales con la suficiente antelación para su adaptación o preparación.

A lo anterior, cabe añadir la constante incorporación de Nuevas Tecnologías orientadas al aprendizaje y a la comunicación entre los alumnos, y entre estos y su profesorado, que también han permitido facilitar el acceso al aprendizaje y a la comunicación de las personas con discapacidad.

\section{La integración en la UNED en cifras}

Para que nos hagamos una idea más clara de la situación plateada, exponemos a continuación los datos descriptivos del alumnado con discapacidad matriculado en la Universidad Nacional de Educación a Distancia. Por ello, recogemos en tablas comentadas los datos relativos al curso 2000-2001 (UNED, 2002), porque eran aquellos los que disponíamos en el momento de realizar el presente artículo.

\section{Descripción de los estudios en los que participan personas con discapacidad (Tabla 1)}

Detectamos que la incidencia en términos absolutos de personas con discapacidad que están matriculadas en la UNED se concentra por estudios en los en tres siguientes: Derecho, Curso de Acceso a mayores de 25 años y Psicología. Estas cifras son un indicativo de en qué facultades/estudios es posible encontrar mayor número de personas integradas. Sin embargo, esta aproximación merece ser matizada en función de las cifras totales de matriculados por Facultad/estudios.

Se puede ver en el gráfico 1 que el peso de cada facultad en la integración de personas con discapacidad en la UNED, esta en relación con el número real de matriculados. Como indicábamos destacan los estudios de Derecho, Psicología y Acceso a Mayores de 25 años. Esta aproximación, aunque limitada, es importante desde el punto de vista de la preparación de las infraestructuras necesarias para facilitar el acceso a la formación y especialmente para la realización de las pruebas escritas, cuando se necesite algún tipo de ayuda específica.

Proporción de la participación de personas discapacitadas por Facultad/estudios (Tabla 2)

Las cifras anteriores sólo pueden valorarse en su justa medida si apreciamos la proporción de personas discapacitadas matriculadas en cada Facultad/estudios. En este caso, se observa en el reparto que las personas con discapacidad se concentran (Tabla 2) en los estu- 
TABLA 1: Alumnado con discapacidad matriculado en la UNED. Curso 2000/01.

\begin{tabular}{|l|c|}
\hline \multicolumn{1}{|c|}{ ESTUDIOS } & TOTAL \\
\hline DERECHO & 436 \\
\hline ACCESO & 351 \\
\hline PSICOLOGÍA & 265 \\
\hline ESCUELA U. DE INFORMÁTICA & 131 \\
\hline ECONÓMICAS Y EMPRESARIALES & 113 \\
\hline GEOGRAFÍA E HISTORIA & 112 \\
\hline POLÍTICAS Y SOCIOLOGÍA & 87 \\
\hline EDUCACIÓN & 61 \\
\hline CIENCIAS & 47 \\
\hline FILOLOGÍA & 45 \\
\hline FILOSOFÍA & 36 \\
\hline E. T. S. DE INGENIEROS INDUSTRIALES & 14 \\
\hline TOTAL ALUMNOS & $\mathbf{1 6 9 8}$ \\
\hline
\end{tabular}

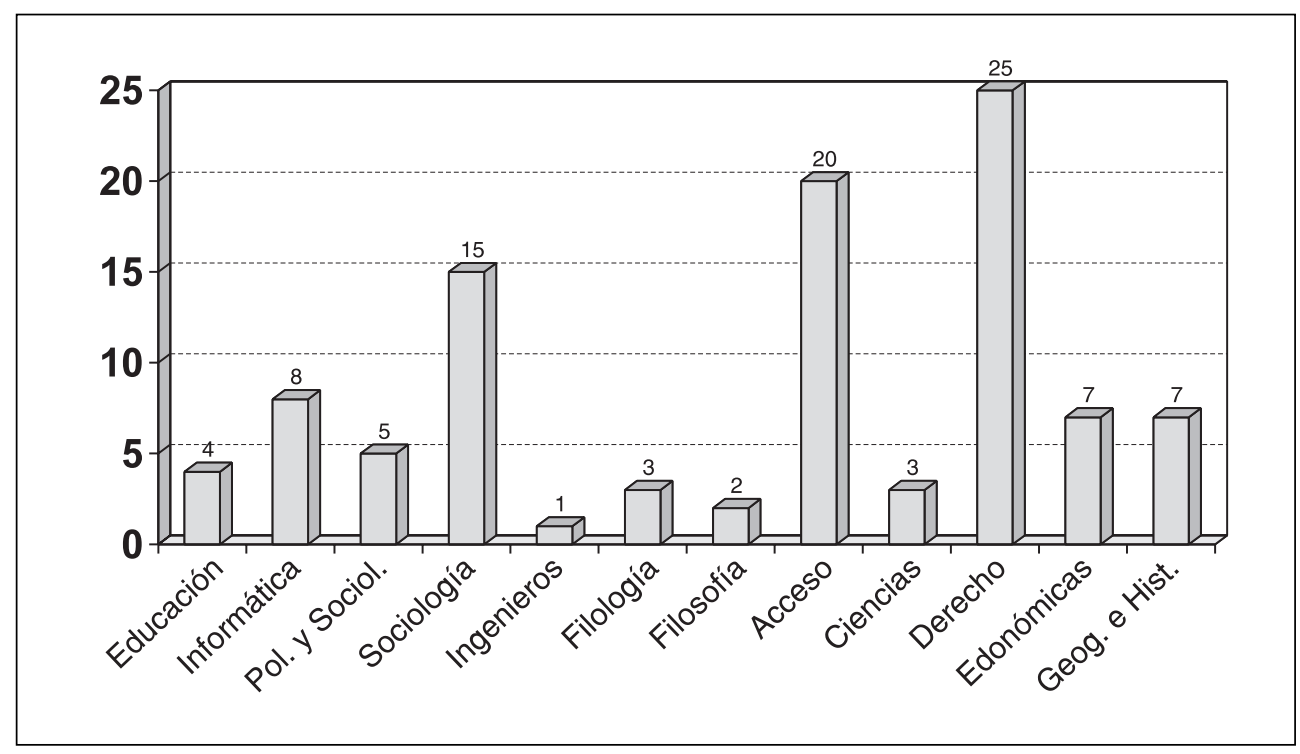

GRÁFICO 1.

Estudiantes con discapacidad por Facultad/estudios en la UNED. 
TABLA 2: Alumnado matriculado en la UNED. Curso 2000/01.

\begin{tabular}{|l|r|r|c|}
\hline \multirow{2}{*}{\multicolumn{1}{|c|}{ ESTUDIOS }} & \multirow{2}{*}{ UNED } & \multicolumn{2}{c|}{ CON DISCAPACIDAD } \\
\cline { 3 - 4 } & & Total & $\mathbf{\%}$ \\
\hline ACCESO & 17817 & 351 & 1,97 \\
\hline FILOLOGÍA & 3183 & 45 & 1,41 \\
\hline FILOSOFÍA & 2557 & 36 & 1,41 \\
\hline DERECHO & 33936 & 436 & 1,28 \\
\hline GEOGRAFÍA E HISTORIA & 9038 & 112 & 1,24 \\
\hline PSICOLOGÍA & 23180 & 265 & 1,14 \\
\hline POLÍTICAS Y SOCIOLOGÍA & 8172 & 87 & 1,06 \\
\hline EDUCACIÓN & 6706 & 61 & 0,91 \\
\hline CIENCIAS & 6126 & 47 & 0,77 \\
\hline INFORMÁTICA & 18214 & 131 & 0,72 \\
\hline ECON. Y EMPRESARIALES & 19166 & 113 & 0,59 \\
\hline E. T. S. ING. INDUSTRIALES & 2836 & 14 & 0,49 \\
\hline NO DETERMINADO & 108 & - & - \\
\hline TOTAL ALUMNOS & $\mathbf{1 5 1 0 3 9}$ & $\mathbf{1 6 9 8}$ & $\mathbf{1 , 1 2}$ \\
\hline
\end{tabular}

dios y titulaciones de: Acceso, Filología, Filosofía, Derecho, Geografía e Historia y Psicología. Ello nos indica que la preferencia de las personas discapacitadas difiere en cierta medida del resto de sus compañeros. Llama especialmente la atención la alta proporción de participación en el Curso de Acceso para mayores de 25 años, lo que interpretamos como una clara preferencia de este tipo de alumnado por la UNED frente a otras vías, normalmente materializadas en pruebas de acceso. Es igualmente significativo que cada 100 alumnos/as matriculados de la UNED, uno es discapacitado.

\section{Desglose por Facultad/estudios y género (Tabla 3)}

En esta tabla (Tabla 3), se aprecia un mayor número de hombres matriculados $(61,19 \%)$ que de mujeres $(38,81 \%)$, con las consabidas excepciones habituales en las carreras tradicionalmente acuñadas como "femeninas": Educación, Psicología y Filología.

Porcentaje de alumnos con discapacidad matriculados en relación con el número total de alumnos de la UNED (Tabla 4)

Recogiendo y analizando los datos sobre los matriculados atendiendo a los tipos de discapacidades, produjimos la tabla 4, en la que se aprecia la predominancia de estudiantes 
TABLA 3: Desglose por género de personas discapacitadas matriculadas en la UNED.

\begin{tabular}{|l|c|c|}
\hline \multicolumn{1}{|c|}{ ESTUDIOS } & HOMBRES & MUJERES \\
\hline CIENCIAS & 37 & 10 \\
\hline DERECHO & 293 & 143 \\
\hline ECONÓMICAS Y EMPRESARIALES & 79 & 34 \\
\hline E. T. S. DE INGENIEROS INDUSTRIALES & 14 & 0 \\
\hline EDUCACIÓN & 16 & 45 \\
\hline FILOLOGÍA & 19 & 26 \\
\hline FILOSOFÍA & 27 & 9 \\
\hline GEOGRAFÍA E HISTORIA & 72 & 40 \\
\hline ESCUELA U. DE INFORMÁTICA & 115 & 16 \\
\hline POLÍTICAS Y SOCIOLOGÍA & 55 & 32 \\
\hline PSICOLOGÍA & 82 & 183 \\
\hline ACCESO & 230 & 121 \\
\hline TOTAL ALUMNOS/AS & 1039 & 659 \\
\hline PROPORCIÓN & $\mathbf{6 1 , 1 9 \%}$ & $\mathbf{3 8 , 8 1 \%}$ \\
\hline
\end{tabular}

TABLA 4: Distribución del alumnado matriculado en la UNED en función del tipo de discapacidad.

\begin{tabular}{|c|c|c|c|c|c|c|c|c|c|c|c|}
\hline \multirow{2}{*}{ ESTUDIOS } & \multicolumn{2}{|c|}{ FÍSICA } & \multicolumn{2}{|c|}{ AUDITIVA } & \multicolumn{2}{|c|}{ VISUAL } & \multicolumn{2}{|c|}{ PSÍQUICA } & \multicolumn{2}{|c|}{ NO DET. } & \multirow{2}{*}{ Totales } \\
\hline & $\%$ & $n^{\circ}$ & $\%$ & $n^{\circ}$ & $\%$ & $\mathbf{N}^{\mathbf{o}}$ & $\%$ & $n^{\circ}$ & $\%$ & $n^{\circ}$ & \\
\hline Ciencias & 70,21 & 33 & 6,38 & 3 & 10,64 & 5 & 10,64 & 5 & 2,13 & 1 & 47 \\
\hline Derecho & 72,48 & 316 & 3,90 & 17 & 13,53 & 59 & 6,19 & 27 & 3,90 & 17 & 436 \\
\hline Econ. Y empres. & 70,80 & 80 & 6,19 & 7 & 20,35 & 23 & 1,77 & 2 & 0,88 & 1 & 113 \\
\hline E. T. S. Ingen. Indust. & 78,57 & 11 & 0,00 & 0 & 14,29 & 2 & 7,14 & 1 & 0,00 & 0 & 14 \\
\hline Educación & 65,57 & 40 & 1,64 & 1 & 22,95 & 14 & 4,92 & 3 & 4,92 & 3 & 61 \\
\hline Filología & 62,22 & 28 & 0,00 & 0 & 24,44 & 11 & 11,11 & 5 & 2,22 & 1 & 45 \\
\hline Filosofía & 63,89 & 23 & 5,56 & 2 & 11,11 & 4 & 16,67 & 6 & 2,78 & 1 & 36 \\
\hline Geografía e hist. & 62,50 & 70 & 1,79 & 2 & 21,43 & 24 & 8,93 & 10 & 5,36 & 6 & 112 \\
\hline Informática & 69,47 & 91 & 12,98 & 17 & 9,92 & 13 & 5,34 & 7 & 2,29 & 3 & 131 \\
\hline Políticas y sociol. & 65,52 & 57 & 3,45 & 3 & 22,99 & 20 & 5,75 & 5 & 2,30 & 2 & 87 \\
\hline Psicología & 68,68 & 182 & 4,53 & 12 & 19,62 & 52 & 4,53 & 12 & 2,64 & 7 & 265 \\
\hline Curso de acceso & 62,68 & 220 & 4,27 & 15 & 19,66 & 69 & 10,54 & 37 & 2,85 & 10 & 351 \\
\hline Totales & 67,79 & 1151 & 4,65 & 79 & 17,43 & 296 & 7,07 & 120 & 3,06 & 52 & 1698 \\
\hline
\end{tabular}


matriculados con discapacidades físicas que suman dos tercios del total. Ello reafirma la observación inicial que hacíamos sobre las posibles causas de participación en la UNED de las personas con algún tipo de discapacidad. Obviamente, una de las dificultades que suelen encontrar en la educación presencial son las barreras físicas de acceso a los propios espacios de formación. La UNED, acaba atendiendo no sólo alumnos/as en la distancia físico-kilométrica sino en otras "distancias" no menos obstaculizantes para el acceso a la formación.

Podemos apreciar más claramente la proporción entre los diferentes tipos de discapacidad en el gráfico 2:

Un aspecto manifiesto (Gráfico 2) es que las discapacidades por determinar representan un bajo porcentaje de estudiantes matriculados en la UNED. Por otro lado, destaca la porción de estudiantes matriculados en esta Universidad vinculados a las de naturaleza sensorial, llevándose la palma las visuales (17\%) frente a las auditivas (5\%). En todo caso, como podemos ver gráficamente el alumnado matriculado con discapacidad física supera a todos los demás grupos $(68 \%)$.

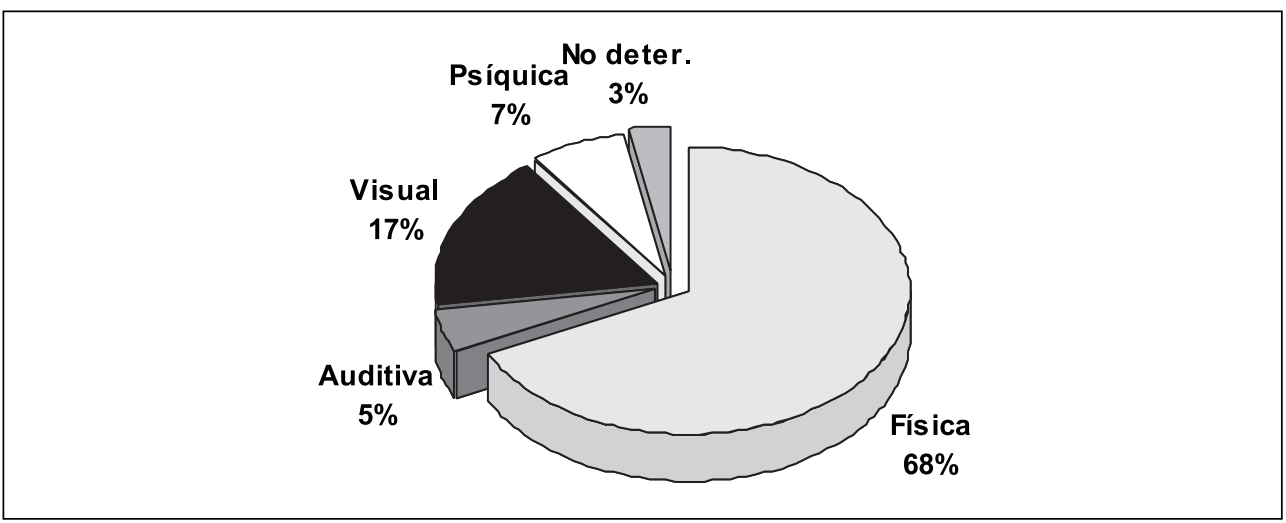

GRÁFICO 2.

Alumnado en la UNED atendiendo a la naturaleza de su discapacidad.

\section{Cuestiones para la reflexión y sugerencias de mejora}

Seguidamente introducimos algunas cuestiones como referentes para una posible reflexión y debate, incorporando asimismo sugerencias dirigidas a la mejora.

\section{¿Qué demandan las personas con discapacidades?}

La UNED no proporciona apoyos específicos para la formación. Normalmente los medios tradicionales son asequibles para la formación de las personas con discapacidad que venimos de describir y, cuando no lo son, deben intentar resolverlo ellos mismos/as. En este papel juegan una función de apoyo fundamental la propia familia y numerosas ONGs. Es de destacar la labor que viene desarrollando la Organización Nacional de Ciegos (ONCE) en la 
búsqueda de soluciones adaptadas a las personas con deficiencias visuales o invidencia. Normalmente, no se suele utilizar el Braille para manuales científicos, por ser éstos de escasa demanda. Para este tipo de peticiones tan puntuales se viene recurriendo a sistemas de grabación en 4 pistas, que se caracterizan por cuadruplicar la duración de un casete convencional o sistema de lectura óptica. Este último, se basa en la aplicación del escáner convencional combinado con un sintetizador de voz que lee convirtiendo el texto escrito escaneado en un mensaje oral. A ello, se unen los mecanismos de aumento más convencionales como la lupa o la telelupa que permite una lectura aumentada a través de pantalla.

Resulta pues difícil de cuantificar y analizar las formas de trabajo de estos alumnos/as salvo a través de un dato del que hay constancia: la solicitud de adaptación que realizan para la prueba presencial. Estas demandas son recogidas y encauzadas por la Unidad de Integración de Alumnos con Discapacidad. Las peticiones más habituales pueden agruparse en:

- Adaptación del tiempo para la realización de la prueba, prolongándolo para posibilitar su realización.

- Adecuación de la prueba: ampliación de letra, examen tipo test, preguntas de examen grabadas o presentadas en Braille.

- Espacio específico: aula aparte.

- Posibilidad de tener un acompañante durante la realización de la prueba.

- Flexibilidad en la utilización de medios técnicos: PC adaptados, máquinas Perkins (escritura Braille), telelupas, etc.

- Apoyo y comprensión del Tribunal.

Sobre estos aspectos podemos añadir que:

- No se permite el aumento del tiempo para la realización de la prueba como consta en el Reglamento para la celebración de pruebas presenciales (UNED, 2000).

- Se suele encontrar muchas reticencias para adaptar los exámenes por procedimientos que suponen la entrega anticipada de la prueba a los servicios que lo van a preparar.

- Se acostumbra a respetar las demandas de aulas aparte para la realización de los exámenes.

- Se autoriza la presencia de un acompañante durante la realización de la prueba siempre que se justifique, ocupándose de la trascripción de las pruebas tipo test, lectura de las preguntas para su grabación, etc.

- No se ponen barreras a la utilización de medios técnicos siempre que los aporte el alumno/a.

- En cuanto al apoyo y comprensión del Tribunal, hay que reconocer que no siempre se consigue en la medida necesaria, pero que también a veces se dan situaciones de demandas abusivas que pueden generar actitudes de desconfianza.

Otro aspecto importante de las demandas se sitúa en el ámbito de los Centros Asociados. Los propios centros reciben peticiones de eliminación de barreras arquitectónicas, mesas y sillas adaptadas en el aula, rampas de acceso, ascensores, servicios adaptados, plazas de aparcamiento reservadas, etc. 


\section{Pautas para el desarrollo de la accesibilidad en la UNED}

A continuación analizamos con detenimiento aspectos que entendemos que se deberían reforzar, crear o mejorar para posibilitar el acceso a la formación de las personas discapacitadas en la UNED, estimando previsiblemente que desencadenarán un mayor nivel de integración en dicha Universidad.

\section{Accesibilidad fisica}

Como se aprecia en las cifras presentadas en el apartado anterior, las personas con discapacidad física suponen el mayor número de los diversos grupos analizados. Es obvio que la UNED está supliendo la falta de accesibilidad y las dificultades de desplazamiento de las personas con este tipo de discapacidad en las modalidades formativas presenciales. Aún así, es de considerar que en la UNED, también es importante la accesibilidad física en las menos frecuentes pero significativas ocasiones en que los alumnos/as deben acudir al centro. Nos estamos refiriendo, especialmente, a la sesión presencial semanal de tutoría en el Centro Asociado y al momento cuatrimestral de la prueba presencial. En ambos casos, nos encontramos aún con barreras que se están intentando subsanar para el libre acceso de las personas con dificultades o imposibilidades motóricas. Estos se hacen más difíciles de resolver al tener uso ocasional los locales, como en el caso con los exámenes cuatrimestrales.

\section{Accesibilidad comunicativa}

Es sabido que la accesibilidad comunicativa es sumamente variada y diversa. Incluimos en ella todas las personas que necesitan ayudas o medios específicos para expresarse o recibir la información, así como, a aquellas que utilizan lenguajes o lenguas diferentes. Tal como hemos descrito en otra ocasión (Feliz y Ricoy, 2002), las personas con estas dificultades o imposibilidades utilizan los Sistema no Habituales de Comunicación Humana, que hemos de tener en cuenta.

De forma específica, en la UNED se utilizan los medios que adaptan, amplían o mejoran los recursos habituales que utilizamos para el aprendizaje, en particular los medios escritos. Sin embargo, las tecnologías de la información y de la comunicación tales como la televisión, el vídeo, la radio, las grabaciones, los espacios en red, la comunicación telemática o el simple teléfono ponen de manifiesto nuevos retos y dificultades que sólo en algunos casos se han resuelto con éxito. Queda mucho trabajo y retos por asumir en este sentido.

\section{Accesibilidad al aprendizaje}

Una cuestión esencial es la accesibilidad al aprendizaje, aspecto en el que la UNED no dio por lo de ahora pasos significativos. Son los propios alumnos/as los que tienen que adaptar sus materiales, contando fundamentalmente con la colaboración de entidades no gubernamentales como la ONCE. 


\section{Formatos clásicos}

Las adaptaciones demandadas más habituales son las de los textos impresos y afectan normalmente a los problemas de la vista (hemos aludido anteriormente a la transcripción en Braille, las grabaciones, las lupas, etc.). En este caso, se tiende a buscar soluciones autónomas en las que el propio alumno/a puede resolver su acceso sin la necesidad de recurrir a la institución (normalmente la ONCE), con la consiguiente exigencia de previsión y disponibilidad de los medios a adaptar. El escáner y el sintetizador de voz son una de las combinaciones más acertadas gracias al apoyo financiero de la citada organización.

\section{Medios audiovisuales}

Los medios audiovisuales concretamente los vídeos, televisión, radio y grabaciones carecen de la adaptación necesaria. En este caso las dificultades pueden ser visuales o auditivas. En estas últimas, es especialmente preocupante la situación al no haber una política de apoyo institucional sólido, ni dentro ni fuera de la UNED. Sería necesario integrar de modo obligatorio en los medios producidos por la UNED las necesarias adaptaciones como los subtítulos o la disposición de transcripciones escritas de las grabaciones.

\section{Recursos virtuales}

Los espacios formativos en red ofrecen nuevas dificultades para las personas con Necesidades Educativas Especiales centradas en el acceso a la información. Se ha de revisar la navegabilidad y la accesibilidad de las páginas y del propio portal de la UNED. No son pocas las dificultades que actualmente ofrecen para un sintetizador de voz o la impresión Braille. Sólo se observan esfuerzos puntuales en disciplinas concretas sin el necesario marco de acción general ${ }^{1}$.

\section{Accesibilidad a la evaluación presencial}

Éste es de los aspectos más apoyados institucionalmente, al estar previsto en dos artículos específicos (artículos 45 y 46) sobre "exámenes en condiciones especiales", del Reglamento sobre Formación de Tribunales y Celebración de Pruebas Presenciales (UNED, 2000). Sin embargo, no son pocas las dificultades que se encuentran para obtener las pruebas con antelación y adaptarlas como en el caso del Braille. Así mismo, la figura de la "persona acompañante" durante el examen carece de la necesaria precisión y se presta a malos usos, interpretaciones o abusos.

\footnotetext{
${ }^{1}$ A modo de ejemplo, puede verse nuestra página abierta de Didáctica General de Educación Social y su plataforma de formación en la adaptación de los cuadernillos de trabajo (www.uned.es/501027, de libre acceso) y de las animaciones introductorias de los temas (virtualdb.uned.es, de acceso restringido a alumnos/as y tutores/as de la asignatura).
} 


\section{La respuesta institucional}

Al observar la demanda de las personas con algún tipo de discapacidad, la UNED creó en el verano del 2000 la Unidad de Integración para Alumnos con Discapacidad (UNED, 2002). Esta Unidad pretende ayudar a los alumnos con discapacidad a través de una ventanilla única, atendiendo todos sus problemas desde el momento de su matrícula. La Unidad es responsable de informar y contactar con todos los sectores que intervienen en la vida formativa de estos alumnos: personal de administración, personal de servicios, profesorado de la Sede Central, y Centros Asociados. Aunque todos deberíamos trabajar para que tengan las mismas oportunidades de formarse que los demás, son muchos los problemas y resistencias que encuentran en el camino.

\section{A modo de conclusión}

Las barreras físicas se han subsanado, en gran medida, tanto en los centros asociados como en la sede central de la UNED. No obstante, la accesibilidad para el alumnado con necesidades educativas especiales al aprendizaje adaptando los materiales es excepcional. Esto en parte, por la ausencia de garantía del apoyo institucional necesario y de un marco de acción general dentro y fuera de la Universidad. Las colaboraciones y los compromisos personales puntuales no garantizan la integración.

En la UNED se matricula un número importante de alumnos con discapacidad. Es con creces la Universidad española con mayor número de alumnado matriculado. No obstante, por el momento no se ha afrontado convenientemente la integración de los mismos a nivel institucional. Quedándose a merced de los alumnos/as o de instituciones externas la posibilidad de adaptación de medios y recursos, así como los apoyos humanos y formativos orientados a la formación.

Aprovechando la creación de la Unidad de Integración para Alumnos con Discapacidad, es necesario que la Universidad Nacional de Educación a Distancia se replantee las directrices y recursos institucionales a disposición de este colectivo de alumnado. Asimismo, tal como hemos sugerido en este trabajo es necesario introducir más medidas de atención especializada desde la propia institución. Esto desde la aportación de respuestas satisfactorias para el alumnado que posibiliten la normalización de sus vidas. Como escenario de formación la Universidad habrá de consolidar a medio plazo mayores oportunidades de integración, poniendo a disposición de los estudiantes los medios y recursos necesarios para responder o adelantarse a las demandas que estos presentan.

\section{Referencias bibliográficas}

Aguilar, L. A. (2000). De la integración a la inclusividad. La atención a la diversidad. Argentina: Espacio Editorial.

Bautista, R. (1993). Educación especial y reforma educativa. Granada: Aljibe.

Best, J. W. (1981). Cómo investigar en educación. Madrid: Morata.

Bisquerra, R. (1989). Métodos de investigación educativa. Barcelona: Ceac. 
Boletín oficial del estado. Ley de Integración Social de los Minusválidos, 13/1982 de 7 de Abril, BOE 103 de 30 de abril de 1982.

Feliz, T. y Ricoy, M. C. (2002). "La atención a la diversidad en el aula: estrategias y recursos". En J. González-Pérez (Coord.), Necesidades educativas especiales e intervención psicopedagógica. (pp. 123-161). Alcalá de Henares: Servicio de publicaciones de la Universidad de Alcalá.

Fox, D. J. (1987). El proceso de investigación en educación. Pamplona: Eunsa.

García Llamas, J. L. (1996). "Investigación correlacional y descriptiva”. En V. García Hoz (Dir.), Problemas y métodos de investigación en educación personalizada. (pp. 307-329). Madrid: Rialp.

López, A. M. y Zafra, M. (2003). La atención a la diversidad en la educación secundaria obligatoria. Barcelona: Octaedro.

Peñafiel, F. (2000). Fundamentos pedagógicos en la intervención de alumnos con necesidades educativas especiales. Granada: Grupo Editorial Universitario.

Moya, A.; Barrero, N. y otros (2000). Enfoque multidimensional de las necesidades educativas especiales. Huelva: Regué Editorial.

Ricoy, M. C. y Feliz, T. (2002). "Consideraciones previas sobre las necesidades educativas especiales". En J. González-Pérez (Coord.), Necesidades educativas especiales e intervención psicopedagógica. (pp. 3-30). Alcalá de Henares: Servicio de publicaciones de la Universidad de Alcalá.

Sánchez, M. P. y Quiroga, M. A. (1998). Perspectivas actuales en la investigación psicológica de las diferencias individuales. Madrid: Ramón Areces.

UNED (2000). Reglamento sobre Formación de Tribunales y Celebración de Pruebas Presenciales. Madrid: UNED.

UNED (2002). Vicerrectorado de alumnado. Unidad de integración de alumnos con discapacidad. Madrid: http://www.uned.es/alumnos-discapacitados/.

Fecha de recepción: $20-2-03$

Fecha de revisión: 5-03-04

Fecha de aceptación: 12-5-04 